Article

\title{
Enhanced Biosensing Activity of Bimetallic Surface Plasmon Resonance Sensor
}

\author{
Ritayan Kashyap ${ }^{1}{ }^{\oplus}$, Soumik Chakraborty ${ }^{1}$, Shuwen Zeng ${ }^{2}{ }^{\circledR}$, Sikha Swarnakar ${ }^{1}$, \\ Simran Kaur ${ }^{3}$, Robin Doley ${ }^{3}$ and Biplob Mondal ${ }^{1, *(\mathbb{D})}$ \\ 1 Department of Electronics and Communication Engineering, Tezpur University, Tezpur 784028, Assam, \\ India; ritayan.kashyap@gmail.com (R.K.); soumik890@gmail.com (S.C.); sikhaswarnakar14@gmail.com (S.S.) \\ 2 XLIM Research Institute, UMR 7252 CNRS/University of Limoges, 123, Avenue Albert Thomas, \\ 87060 Limoges CEDEX, France; shuwen.zeng@unilim.fr \\ 3 Department of Molecular Biology and Biotechnology, Tezpur University, Tezpur 784028, Assam, India; \\ simran@tezu.ernet.in (S.K.); doley@tezu.ernet.in (R.D.) \\ * Correspondence: biplobm@tezu.ernet.in; Tel.: +91-3712-275266; Fax: +91-3712-267005
}

Received: 18 July 2019; Accepted: 10 October 2019; Published: 21 October 2019

\begin{abstract}
Surface plasmon resonance (SPR) sensors present a challenge when high sensitivity and small FWHM (full width at half maximum) are required to be achieved simultaneously. FWHM is defined by the difference between the two extreme values of the independent variable at which the value of the dependent variable is equal to half of its maximum. A smaller value of FWHM indicates better accuracy of SPR measurements. Theoretically, many authors have claimed the possibility of simultaneously achieving high sensitivity and small FWHM, which in most of the cases has been limited by experimental validation. In this report, an experimental study on the improved surface plasmon resonance (SPR) characteristics of gold over silver bimetallic sensor chips of different film thicknesses is presented. A comparative study of antigen-antibody interaction of the bimetallic chip using a custom-made, low-cost, and portable SPR device based on an angular interrogation scheme of Kretschmann configuration is performed. Pulsed direct current (DC) magnetron-sputtered bimetallic films of gold over silver were used in the construction of the SPR chip. The FWHM and sensitivity of the bimetallic sensors were firstly characterized using standard solutions of known refractive index which were later immobilized with monoclonal anti-immunoglobulin $\mathrm{G}(\mathrm{IgG})$ in the construction of the SPR biochip. Spectroscopic measurements such as ultraviolet-visible light spectroscopy (UV-Vis) and Fourier-transform infrared spectroscopy (FTIR) were used for the confirmation of the immobilization of the antibody. The performance of the bimetallic SPR biochip was investigated by exposing the sensor to various concentrations of the target protein. The results indicated that the bimetallic sensors of silver/gold had a 3.5-fold reduced FWHM compared to pure gold-based sensors, indicating a higher detection accuracy. In addition, they exhibited a significant shift in resonance angle as high as $8.5 \pm 0.2$ due to antigen-antibody interaction, which was $\sim 1.42$-fold higher than observed for pure silver-based sensors.
\end{abstract}

Keywords: full width at half maximum (FWHM); monoclonal anti-IgG; pulsed DC magnetron; sensitivity; surface plasmon resonance

\section{Introduction}

Over the past few years, surface plasmon resonance (SPR) has emerged as an important real-time and label-free optical detection technique used to measure changes in the refractive index near the sensor surface. The use of SPR sensors for monitoring processes at metal interfaces was recognized in the late 1970s [1]. Nylander et al. first demonstrated the use of SPR for gas detection and biosensing in 
1982 [2-4]. The principle of SPR involves the excitation of electron density oscillations called surface plasmon waves (SPWs) when p-polarized light is incident on a metal-dielectric interface under the condition of total internal reflection. When the energy and the momentum of the incident light match with the SPW, it gives rise to the condition of resonance, resulting in a reduced intensity of the reflected light. The condition of resonance depends on the wavelength of the light source, the angle of incidence, and refractive indices of the metal and dielectric, as well as the thicknesses of the metal and adhering layers. In the angular interrogation method, the condition of resonance is observed as a sharp fall in signal intensity at a specific angle of incidence, whereas, in the wavelength interrogation method, the same is observed at a specific wavelength of light. Any change in refractive index at the sensor surface is detected in the form of a shift in the SPR dip at the output [5,6].

Over the past few years, several generations of instrumentation for SPR biosensing were witnessed, ranging from prism [7-10] and grating-coupled [11] instrumentation to fiber-coupled [12,13] SPR sensing. Light is passed through the sample solution in the case of a grating-coupled system, which results in a poor signal from the sensor. Although fiber-optic-based configurations were devised for the purpose of flexibility and miniaturization, prism-based SPR sensors still offer better stability and sensitivity [13].

The choice of the metal layer and its film thickness has a significant impact on the characteristics of the SPR sensor. Preferably, metals like silver and gold are used because of their ability to resonate conduction-band electrons at a suitable wavelength, in addition to their thermal and biochemical stability, their inert behavior, and the ease of attachment of organic molecules to their surface. Silver is cheaper than gold, and a thin film of silver with a thickness of around $50 \mathrm{~nm}$ is characterized by a sharp SPR signal, leading to error minimization in determining resonance angle [14]. The sharpness of the resonance curve occurs due to the lower imaginary value of the dielectric constant of silver than gold at a given visible wavelength [15]. In addition, a surface plasmon wave (SPW) which propagates along the surface of silver is attenuated to a lesser extent and exhibits higher localization of the electromagnetic field in the dielectric compared to an SPW supported by gold [13]. A 50-nm-thick silver film has an enlarged penetration depth of $219 \mathrm{~nm}$ for light with a wavelength of $630 \mathrm{~nm}$, compared to only $164 \mathrm{~nm}$ for a gold film of the same thickness [16]. However, silver is unstable and prone to oxidation in ambient air. Gold, on the other hand, is not only chemically stable but also produces a larger shift in resonance angle than silver, corresponding to the changes in refractive index of the sensing layer [17]. Gold, with a higher real value of dielectric constant than silver, demonstrates a higher shift in the resonance dip due to the change in refractive index of the sensing layer [15]. A bimetallic film of silver/gold combines the advantages of both silver and gold, representing its superior candidature for SPR sensors. Zynio et al. were the first to propose bimetallic films of gold and silver, with gold being deposited on top of silver, to study SPR [18]. It was reported that the bimetallic films displayed a large shift in resonance angle due to the changes in ambient refraction index similar to gold films, as well as showing a narrow resonance curve similar to silver, thereby leading to a high signal/noise ratio. In addition, the silver was protected against oxidation by the outer gold layer. Yuan et al. presented a theoretical and experimental study on the sensitivity and stability optimization of bimetallic silver/gold SPR sensors. The experimentally obtained values of FWHM for gold, silver, and bimetallic silver/gold sensors in air were reported to be $10.67^{\circ}, 0.71^{\circ}$, and $2.22^{\circ}$, respectively, which indicate a great reduction in the FWHM for the bimetallic sensor compared to gold [19]. However, the corresponding value of FWHM for a pure silver film is slightly better than that of the bimetallic film configuration. The authors also optimized the film thickness for maximum evanescent field enhancement in bimetallic SPR sensors based on a numerical and experimental study [20]. The bimetallic configuration exhibited sharper FWHM, which was about three times narrower than the single gold film configuration, as well as higher stability and better adherence of biomolecules compared to single silver film configuration. A theoretical study of the improvement in sensitivity of SPR sensors with silver/gold bimetallic films was done by $\mathrm{Wu}$ and $\mathrm{Hu}$ [21]. Chen et al. used a bimetallic configuration of silver/gold in order to improve the sensitivity and stability of a commercial SPR instrument [22]. The bimetallic film was 
also utilized to improve the sensitivity of an SPR optical fiber sensor by Huang et al. [23]. In 2016, Chen and Lin presented a theoretical study of silver/gold bimetallic SPR sensor chips with both high sensitivity and low FWHM, based on the Kretschmann configuration angular interrogation scheme [24]. The optimized film thickness of silver/gold was analyzed for air and water, and it was reported that the bimetallic sensor chips showed a higher sensitivity than the single silver-based one and a lower FWHM than the gold-based one. Many authors reported the possibility of individually achieving high sensitivity or small FWHM using bimetallic silver/gold SPR sensor chips. However, very few detailed reports are available on the experimental validation of simultaneously achieving reasonably high sensitivity and small FHWM.

In this work, we present a detailed literature review of bimetallic silver/gold SPR sensor chips, as well as an experimental study to optimize film thickness and composition in order to simultaneously achieve reasonably high sensitivity and small FWHM, thereby establishing a trade-off between the two. The SPR biosensors were constructed by immobilizing the sensors with a monoclonal antibody against the immunoglobulin (IgG) protein. The performance of the SPR biosensors based on a bimetallic film of silver and gold was compared with that of sensors based on pure silver or gold.

\section{Experimental Methods}

\subsection{Chemicals and Materials}

Silver and gold coins (99.9\% pure) measuring 2 inches in diameter were purchased from the State Bank of India, a public sector banking and financial services company in India. N-hydroxysuccinimide (NHS), 1-ethyl-3 (3-dimethylaminopropyl) carbodiimide (EDC) and 11-mercuptoundecanoic acid (11-MUA) were procured from Sigma-Aldrich. The Monoclonal anti mouse protein (IgG2b) was procured from Invitrogen. The rest of the chemicals of analytical grade were purchased from Sigma-Aldrich.

\subsection{Sensor Fabrication}

Metal-coated microscopic cover slips $(18 \mathrm{~mm} \times 18 \mathrm{~mm} \times 1 \mathrm{~mm})$ with a refractive index of 1.515 (at $632.8 \mathrm{~nm}$ wavelength) were used as substrates for the construction of the SPR sensor. Deposition of metal film over the cover slip was done by Pulsed DC Magnetron Sputtering using a Planar DC Magnetron Sputtering Unit (Model No 12 MSPT, Hind High Vacuum Company Private Limited). A thin layer of chromium $(\sim 5 \mathrm{~nm})$ was used to improve the adhesion of gold with glass. Prior to deposition, the cover slips were rinsed in acetone vapor, methanol and De-Ionized (DI) water. To construct SPR biochip surface of the metal films were bio-functionalized with suitable antibodies using amine coupling chemistry [25]. The methodology followed for the covalent immobilization of antibody on the metal-coated substrate is shown in Figure 1. Rusmini et al. described in depth the various schemes involved in the immobilization of protein [26]. A strong affinity towards the noble metal surfaces is exhibited by the thiol groups which allow the formation of a covalent bond between the metallic atom and the sulphur head group of thiol [27-29].

Firstly, a self-assembled monolayer (SAM) of 11 MUA $(2-3 \mathrm{~nm})$ was formed to functionalize the metal-covered glass slides by immersing them in anethanoic solution of 11-MUA (molarity $1 \mathrm{mM}$ ). Proper molecular organization was achieved by dipping the slides for $12 \mathrm{~h}$ at room temperature in a tight-lid container. Next, the MUA-coated chips were thoroughly rinsed with ethanol and dried under nitrogen stream. MUA acts as a linking agent with its sulphur head, forming a thiolate with the metal crystal lattice, leaving the carboxylic-functional group free to link with other functional group in successive steps. In the following step, a micropipette was used to successively deposit separate solutions of EDC $(75 \mathrm{mM})$ and NHS $(25 \mathrm{mM})$ followed by incubation in a tight-lid petri dish for $1 \mathrm{~min}$. Further, the slides were immersed into monoclonal anti-mouse IgG2b solution $(2 \mathrm{mg} / \mathrm{mL})$ for $2 \mathrm{~h}$ to form stable monomolecular layer. The experiment was performed in $50 \mu \mathrm{L}$ PBS buffer to maintain a $\mathrm{pH}$ of 7.4. In order to prevent the undesirable linkage of the free amine functional group from EDC-NHS to 
the target analytes, the chips were dipped in $50 \mathrm{mM}$ ethanolamine $(100 \mu \mathrm{L})$ solution for $20 \mathrm{~min}$. Lastly, the chips were gently cleaned using $0.5 \mathrm{~mL}$ phosphate buffer solution, and a storage temperature of $4{ }^{\circ} \mathrm{C}$ was maintained for $12 \mathrm{~h}$ prior to performing SPR measurements.

A set of four sensors were prepared by varying the type of metal and film thickness: two consisting of pure silver/gold film and the other two with silver on gold bimetallic films. The samples and their specifications are indicated in Table 1. The rate of deposition of silver and gold were set to $0.226 \mathrm{~nm} / \mathrm{s}$ and $0.2987 \mathrm{~nm} / \mathrm{s}$ respectively. The thickness of the metal was chosen based on the fact that: (a) a film thickness of $50 \mathrm{~nm}$ for metal is suited for maximum electric field enhancement, producing superior resonance condition in SPR spectroscopy as reported by S. Mahapatra et al. and Kuo-Chi Chiu et al. [30,31]; (b) a combined thickness of $50 \mathrm{~nm}$ for the bimetallic films of silver and gold with the gold thickness comprising between $12-25 \mathrm{~nm}$ is reported to protect the silver layer from oxidation [18-20]. X-ray reflectivity (XRR) method was used for the film thickness measurement [32]. Finally, all the samples were immobilized with anti-IgG for the construction of the SPR biochip.

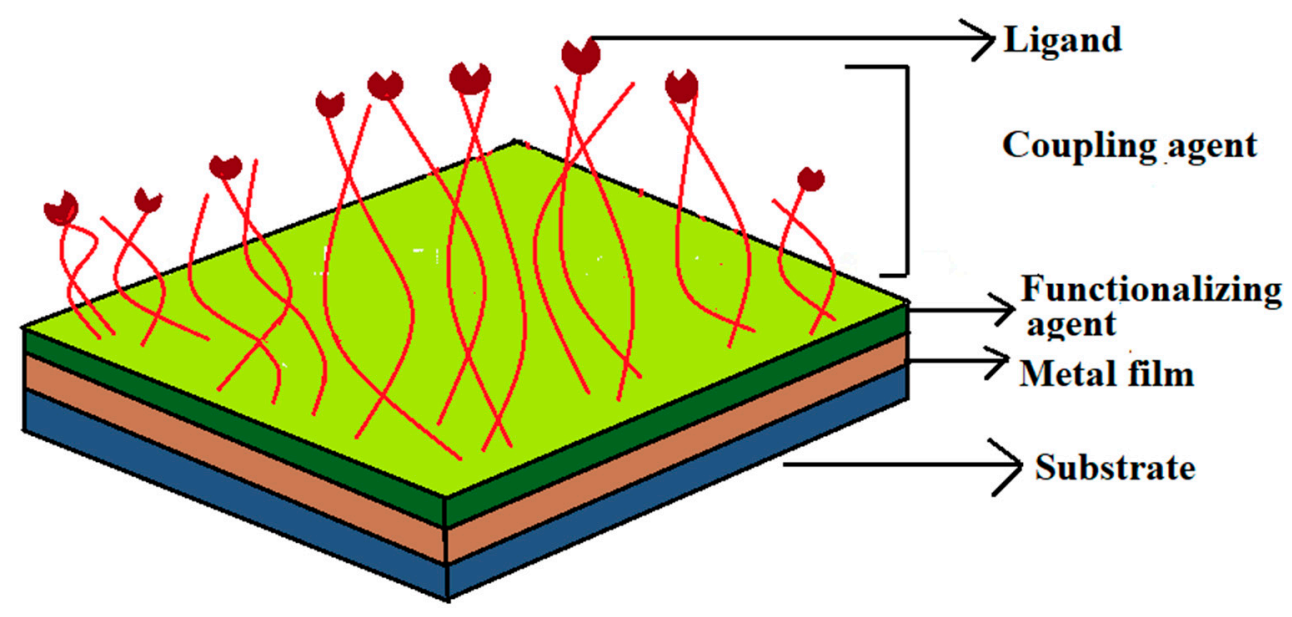

Figure 1. Representation of protein immobilization scheme.

\subsection{Method of Elemental Characterization}

The covalent binding of the antibody with the metal surface is confirmed by spectroscopic measurements. Ultraviolet-visible light spectroscopy (UV-Vis) optical absorption spectrometer (Shimadzu-2000) was used to record the adsorption spectra of the samples prior and post protein immobilization in the range $200-900 \mathrm{~nm}$. Analysis of the chemical structures post-deposition of different layers over the sensor surface was done by Fourier transform infrared spectroscopy (Nicolet Impact 410).

\subsection{SPR Device and Measurements}

Kretschmann's configuration-based optical transduction method was utilized for converting change in the refractive index at the sensor surface into a useful electrical signal. An inexpensive custom-made measurement setup was fabricated for this purpose comprising of a BK7 grade prism mounted with two supports from two sides of the prism. Such a provision for easy mounting and removal of the prism was kept for cleaning of the prism and sensor head as and when required. A laser diode $(632.8 \mathrm{~nm}, 5 \mathrm{~mW})$ was mounted on a "L"-shaped leg and its movement was incremented in steps using a servomotor to adjust the incident angle of the incoming light. The angular span of the incident beam to the sensor surface was $45-80^{\circ}$. The incoming light from the laser diode was made to pass through a polarizer in order to produce p-polarised light and then made to fall on the sensor surface at different angles of incidence. In order to avoid the presence of any air gap, an index-matching fluid was used to stick the microscopic cover slips with the glass prism (BK7).The device also consists of a specially designed flow cell (approximately $280 \mathrm{~mm}^{3}$ ) regulated by a peristaltic pump to pass 
the stream of test samples through the sensor surface controlled using an Arduino microcontroller. The flow cell can be readily attached to the sensor surface using O-ring to prevent leakage of test fluid. The test samples were made to flow through the four sets of sensor surfaces individually. The detector array of the device comprises of parallel-connected LDRs (Light Dependent Resistors) from which the data is fed into the Data Acquisition system. One of the key distinctive features in the design of the device lies in the positioning of the light source and the prism, thereby eliminating the requirement of any rotary stage for placing the prism and consequent passage of test samples over it as reported by Maharana et al. and Ong et al. thus reducing possibility of leakage of samples [15,20]. The whole arrangement measures $25 \times 25 \times 20 \mathrm{~cm}^{3}$ with an approximate weight of $500 \mathrm{gm}$ and provides a fast, selective and label-free method to study Surface Plasmon Resonance Spectroscopy. Figure 2a shows the schematic representation of the experimental set-up.

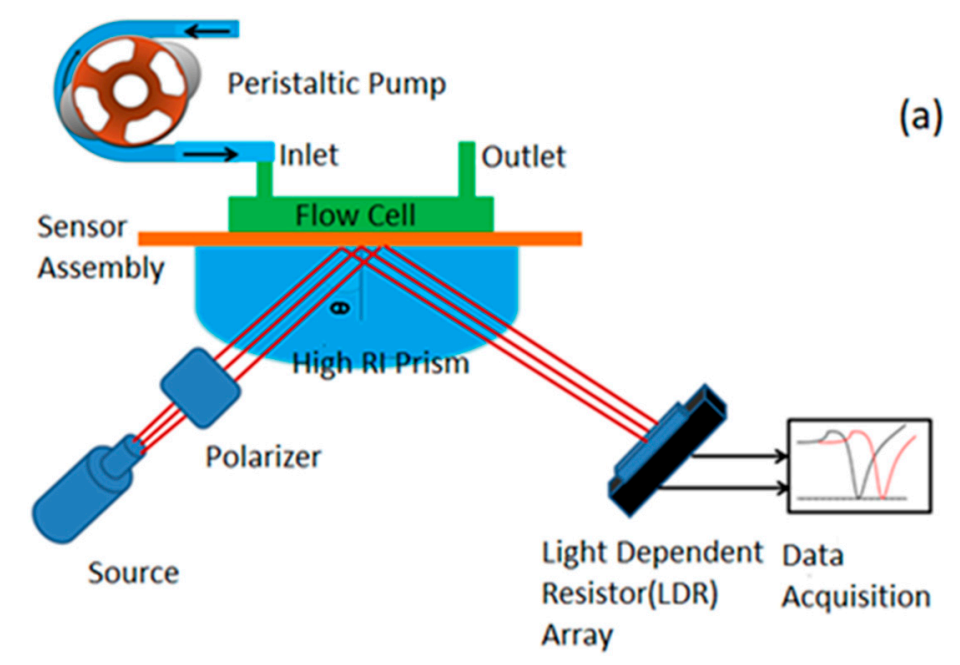

(b)

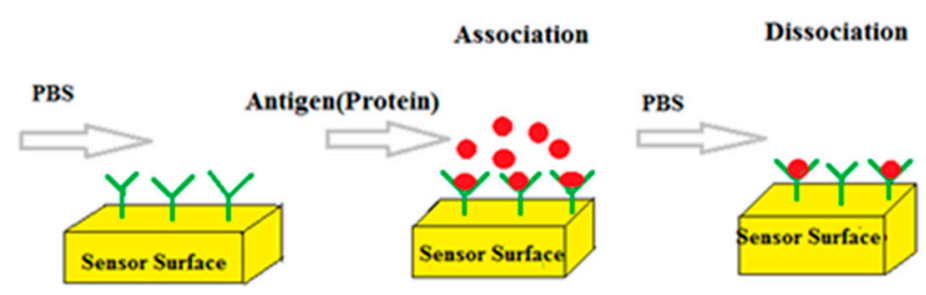

Figure 2. Representation of (a) the experimental set-up and (b) sensor measurement procedure.

Initially, phosphate buffer solution (PBS) ( $\mathrm{pH}$ 7.4) was flowed over the self-assembled monolayer so that a baseline reading was established before studying the binding of protein. Thereafter, different concentrations of protein IgG $(0.1-1.0 \mathrm{mg} / \mathrm{mL})$ prepared by dissolving in the PBS solution were passed over the sensor surface for $10 \mathrm{~min}$ resulting in the association of protein in the test sample with monoclonal anti-IgG as shown in Figure $2 \mathrm{~b}$. Lastly, in order to carry out the dissociation of protein with the antibody, a stream of PBS was flowed over the sensor surface.

Table 1. Sample and Specifications.

\begin{tabular}{cccc}
\hline Sample ID & Deposited Metal Film & Film Thickness (nm) & Deposition Time (s) \\
\hline Ag-I & Silver $(\mathrm{Ag})$ & 50 & 221 \\
\hline $\mathrm{Au}-\mathrm{II}$ & Gold $(\mathrm{Au})$ & 50 & 167 \\
\hline $\mathrm{Ag}-\mathrm{Au}-\mathrm{III}$ & Silver-Gold $(\mathrm{Ag}-\mathrm{Au})$ & $35(\mathrm{Ag})-15(\mathrm{Au})$ & $\mathrm{Ag:} 154 \mathrm{Au}: 50$ \\
\hline $\mathrm{Ag}-\mathrm{Au}-\mathrm{IV}$ & Silver-Gold $(\mathrm{Ag}-\mathrm{Au})$ & $30(\mathrm{Ag})-20(\mathrm{Au})$ & $\mathrm{Ag}: 132 \mathrm{Au}: 67$ \\
\hline
\end{tabular}




\section{Results}

\subsection{Elemental and Structural Characterization}

The immobilization protocol for SPR sensor involves a number of steps and the sensor surface consists of a number of layers stacked together. The surface composition and structure of the deposited layers viz., thickness of the metal layer, and morphology of the SAM layer (applied for surface functionalization) and the NHS-EDC (applied for surface activation) are essential to ensure the immobilization of a properly oriented antibody. Spectroscopic measurements such as ultraviolet-visible light spectroscopy (UV-Vis) and Fourier-transform infrared spectroscopy (FTIR) were performed to confirm the proper immobilization of the antibody.

UV-Vis spectroscopic optical absorbance spectra of the sensor surface pre- and post-antibody immobilization with an underlying $50 \mathrm{~nm}$ silver film is presented in Figure 3. The absorbance peak occurs at $430 \mathrm{~nm}$ for pure silver and the subsequent shifts occur due to deposition of other successive layers on the silver film. The chemisorption of thiol molecules to the surface of the metal due to functionalization of 11-MUA, amine-based coupling chemistry with carboxylated silver using EDC-NHS, and finally antibody conjugation leads to a red-shift in the absorption peak by $20 \mathrm{~nm}, 39 \mathrm{~nm}$ and $21 \mathrm{~nm}$ respectively. The corresponding absorbance peaks were observed at $450 \mathrm{~nm}, 489 \mathrm{~nm}$ and $510 \mathrm{~nm}$ respectively in the UV-Vis spectroscopy. The addition of molecular layers causing red shift of the absorbance peaks is indicative of an increment in the local dielectric constant close to the surface of the metal. Aslan et al. and Weng et al. also reported similar results [33,34].

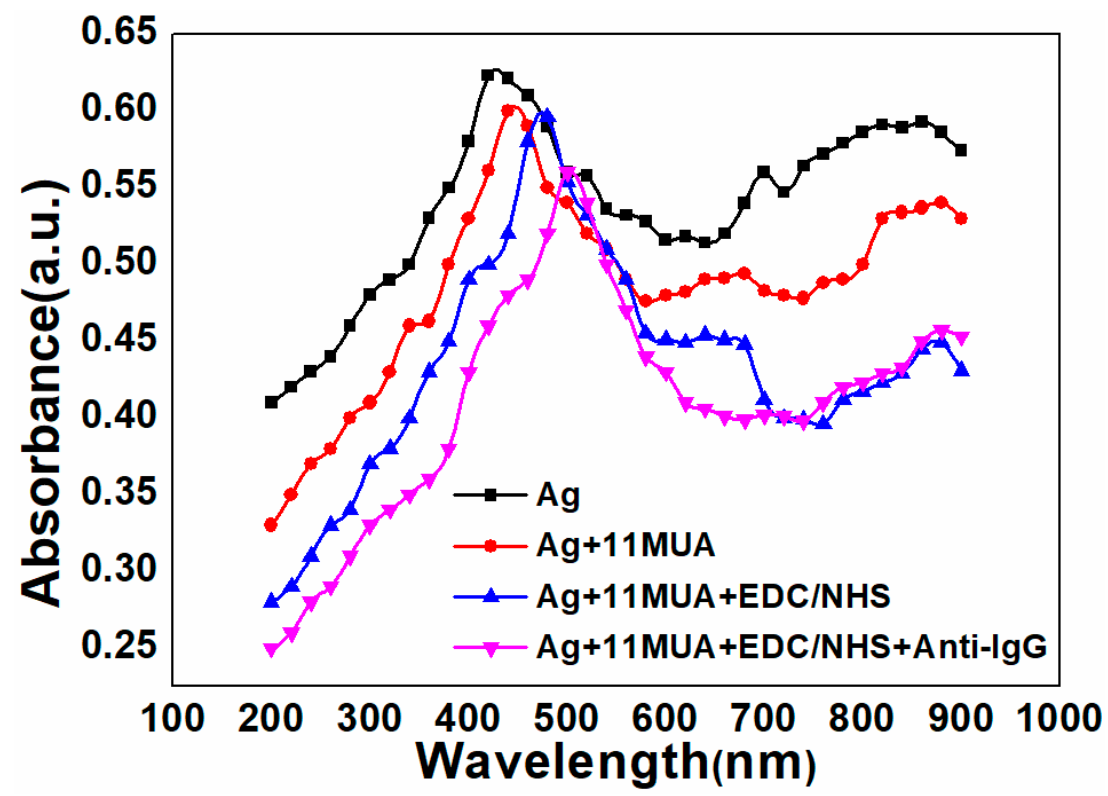

Figure 3. UV-Vis spectroscopic optical absorbance spectra of the sensor surface before and after immobilization of antibody.

The infrared spectroscopy (IR) spectrum of the sensor surface post deposition of the 11MUA, EDC-NHS and anti-IgG are shown in Figure 4. The end functional group of 11-MUA being $\mathrm{COOH}$, the amine functional group of NHS binds with the COOH group through EDC to get converted into the end group COO-NHS. On antibody immobilization (anti IgG), this end group finally changes into COO-N-IgG. The region of carbon band in the IR spectra is shown in Figure $4 \mathrm{a}$. It is characterized by two absorbance peaks that appear at the wave numbers $2850 \mathrm{~cm}^{-1}$ and $2925 \mathrm{~cm}^{-1}$ occurring because of $\mathrm{CH}_{2}$ symmetric stretch and $\mathrm{CH}_{2}$ asymmetric stretch. The region of ester, carboxyl and amide in the IR spectra is shown in Figure $4 \mathrm{~b}$. The peaks appearing at $1740 \mathrm{~cm}^{-1}$ and $1680 \mathrm{~cm}^{-1}$ confirm ester and amide bond formation between 11-MUA with NHS. The peaks at $1820 \mathrm{~cm}^{-1}$ and $1300 \mathrm{~cm}^{-1}$ are due to the stretch modes in the COO-NHS ester. Further, the characteristic bands observed at $1588 \mathrm{~cm}^{-1}$ and 
$1681 \mathrm{~cm}^{-1}$ show the formation of new amide bonds which confirm the immobilization of anti-IgG on the surface.
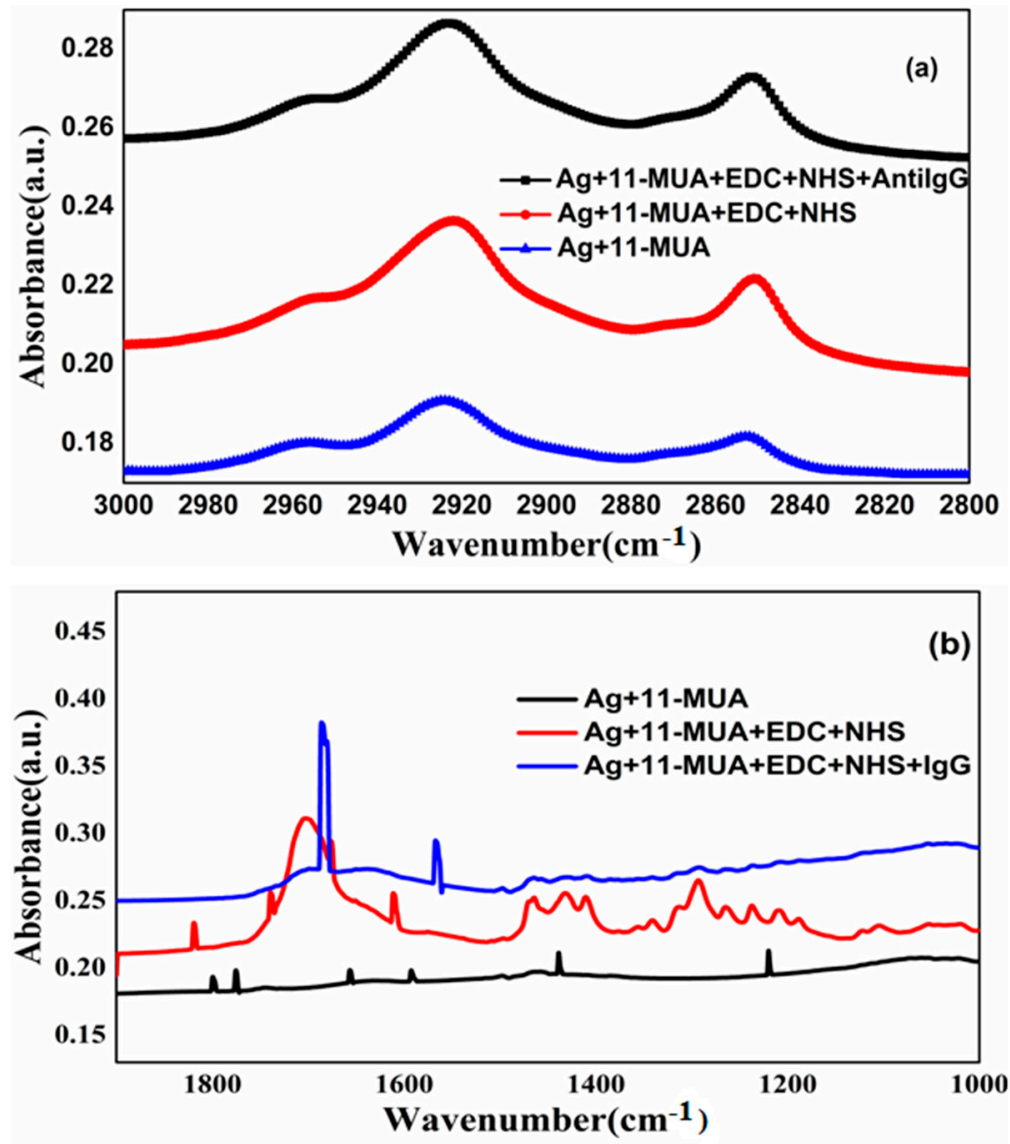

Figure 4. Transform Infrared spectra of the sensor surface before and after the immobilization of antibody: (a) absorbance vs wavenumber $\left(2800-3000 \mathrm{~cm}^{-1}\right.$ ), (b)absorbance vs wavenumber (1000-1800 $\left.\mathrm{cm}^{-1}\right)$.

\subsection{Sensor Response}

Before performing a core study to evaluate the behavior and interaction capacity of the fabricated biosensor to monoclonal antibody, the performance of the custom-made measurement system was established by measuring SPR data against standard solutions of the known refractive index. For this purpose, water, acetone and hexane with refractive indexes of 1.3317, 1.3578 and 1.3744 (at $632.8 \mathrm{~nm}$ ) respectively were passed through the pure metal surface of the sensors without any protein immobilized on them. The SPR data recorded at room temperature is presented in Figure 5 showing the variation of reflected light intensity with angle of incidence for the four different sensors as mentioned in Table 1. The shape of the SPR curve is strongly influenced by the type of metal layer in contact with the test sample. All the sensors show identical behavior of shifting of the resonance dip towards a higher angle of incidence as the refractive index of the test sample increases. The figure also indicates that the sensor with the bare silver film produces the sharpest absorption peak (resonance dip) followed by the sensors constructed with bimetallic film of silver-gold and bare gold film. However, it may be noticed that the bimetallic films produce resonance spectra characteristics close to that of bare silver film. The characteristics of the curve like its shape, full width at half maxima (FWHM), position of the SPR dip etc., signify important meaning in sensing applications. A smaller value of FWHM implies a narrow curve, signifying better accuracy of measurements. FWHM values were extracted from the measured data for each sensor, and the obtained results are summarized in Table 2. Four- to six-fold reductions in FWHM are achieved with bimetallic sensors over pure gold-based ones. Since servo motors have 
relatively poor accuracy, for each sensor, five sets of measurements were recorded with individual test samples (water/acetone/hexane). Error value $( \pm$ ) indicates the maximum deviation of the successive measurements among a set of five measurements. FWHM values were calculated by measuring the width of the characteristic SPR curve at the mid-point of the two extreme y-coordinate values.
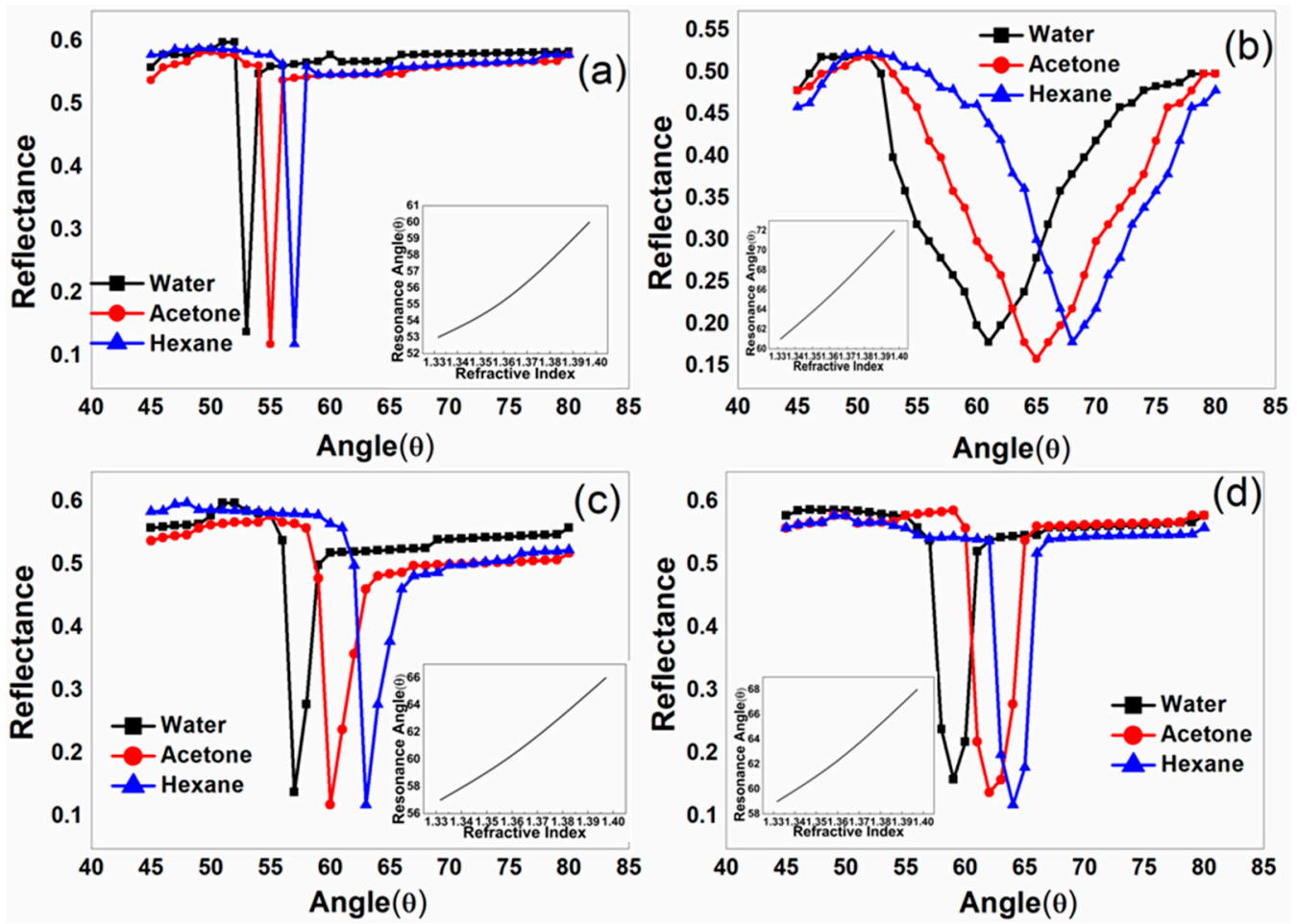

Figure 5. Data against standard test samples of water, acetone and hexane for sensors (a) Ag-I (b) Au-II (c) Ag-Au-III (d) Ag-Au-IV. Embedded figure plots the changes of resonance angle with the refractive index of the medium. Corresponding to a higher change in refraction index of the sample, the resonance happened at a higher angle of incidence.

Table 2. Experimentally Measured FWHM for Different Sensors.

\begin{tabular}{ccccc}
\hline \multirow{2}{*}{ Sample } & \multicolumn{4}{c}{ FWHM $\left(^{\circ}\right)$} \\
\cline { 2 - 5 } & Ag-I & Au-II & Ag-Au-III & Ag-Au-IV \\
\hline Water & $1 \pm 0.1$ & $12 \pm 0.3$ & $2 \pm 0.2$ & $3 \pm 0.2$ \\
\hline Acetone & $1 \pm 0.2$ & $12 \pm 0.3$ & $2.5 \pm 0.1$ & $3.5 \pm 0.3$ \\
\hline Hexane & $1 \pm 0.1$ & $11 \pm 0.2$ & $2 \pm 0.1$ & $3 \pm 0.2$ \\
\hline
\end{tabular}

The sensitivity of an angular interrogated SPR sensor is determined by the corresponding shift of the resonance dip in degrees when there is a change in the refractive index of the sensing layer. Sensitivity is said to be high if for a small change in refractive index the shift in the resonance dip is large [35].Table 3 lists the experimental shift of the resonance angle (in degrees) due to a change in the refractive index of the sensing layer, calculated from the graphs in Figure 5. From the table, it is seen that for a change in refractive index of 0.0261RIU (due to Water and Acetone), gold sensor chip shows the highest shift of resonance angle due to a change in the ambient refraction index while the same is the least for silver. The bimetallic sensor chips also exhibit a significant high shift of resonance angle in comparison to that of silver sensor chip, indicating relatively good sensitivity which is better than that reported by Yuan et.al. [19] and Chen and Lin [24]. 
Table 3. Shift in Resonance Angle for the Sensors due to a Refractive Index Change of 0.0261 RIU.

\begin{tabular}{ccccc}
\hline \multirow{2}{*}{$\begin{array}{c}\text { Refractive Index } \\
\text { Unit (RIU) }\end{array}$} & \multicolumn{4}{c}{ Shift of the Resonance Angle $\left(^{\circ}\right.$ ) } \\
\cline { 2 - 5 } & Ag-I & Au-II & Ag-Au-III & Ag-Au-IV \\
\hline 0.0261 & $2 \pm 0.1$ & $4 \pm 0.3$ & $3.5 \pm 0.1$ & $3.6 \pm 0.2$ \\
\hline
\end{tabular}

Figure 6 shows variation in intensity of the light reflected from the sensor chip (Ag-I) with angle of incidence. Protein IgG with a concentration of $0.1 \mathrm{mg} / \mathrm{mL}$ is flowed over the monoclonal anti-IgG immobilized sensor chip Ag-I. The bonding among the stacked layers is confirmed by the shift in the resonance dip due to the successive deposition of additional layers on the surface of the sensor.

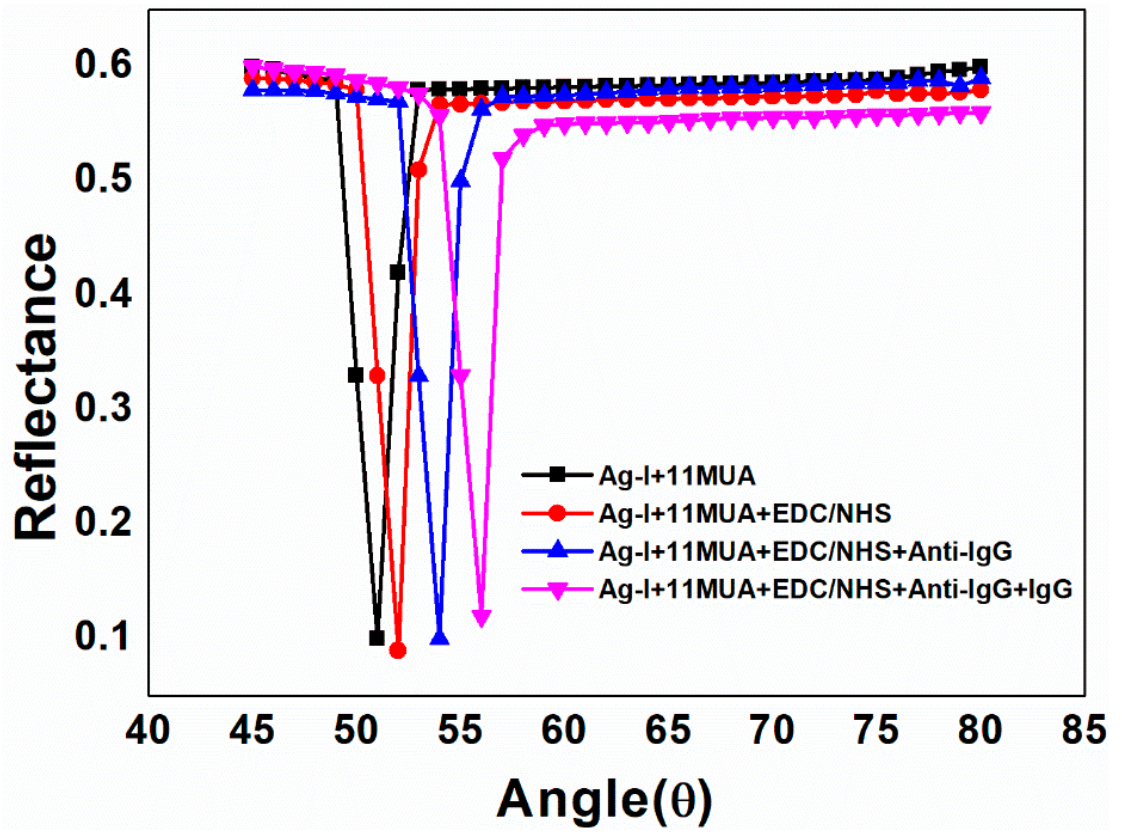

Figure 6. Reflectance vs. incident angle of stacked layers (test sensor: Ag-I test protein concentration: $0.1 \mathrm{mg} / \mathrm{mL})$.

The resonance spectra of the four sensors when they were exposed to different concentrations $(0.1 \mathrm{mg} / \mathrm{mL}, 0.4 \mathrm{mg} / \mathrm{mL}, 0.7 \mathrm{mg} / \mathrm{mL}$ and $1.0 \mathrm{mg} / \mathrm{mL})$ of antibody protein (IgG) is plotted in Figure 7 . It is evident that the SPR characteristic curve is not much affected by the change in concentration of the sample but changes with a change in the underlying metal film. As can be seen from Table 4, the FWHM of the curves remain nearly equal for each sensor when the concentration of sample is varied in the range $0.1-1.0 \mathrm{mg} / \mathrm{mL}$. However, a significant variation in FWHM can be identified when the underlying metal is changed for each concentration of the test sample. The obtained results indicate a 2.5 to 3.5-fold reduction in FWHM with bimetallic sensors compared to that of pure gold-based ones which is better than that reported by Ong et al. [20]. The smaller value of FWHM for bimetallic sensors will significantly improve the accuracy of measurement. The flow of protein (antigen) of higher concentration results in a higher degree of refractive index change in the medium close to the sensor interface due to a higher binding event between antigen-antibody. From the data in Table 4, it is evident that the sensor with the gold interface produces the highest resonance shift of $9 \pm 0.2$ due to antigen protein with a concentration of $1.0 \mathrm{mg} / \mathrm{mL}$ in reference to the buffer solution. For bimetallic sensors, the shift is relatively higher than silver and close to the gold interface-based sensor. This is also evident from Figure 8, where the resonance angle of SPR is plotted against the concentration of antigen protein for the four sensors. The results confirm the fact that with the increase of thickness of the underlying silver film in the bimetallic sensor chip, both the FWHM and the sensitivity decrease as reported by Chen and Lin [24]. 

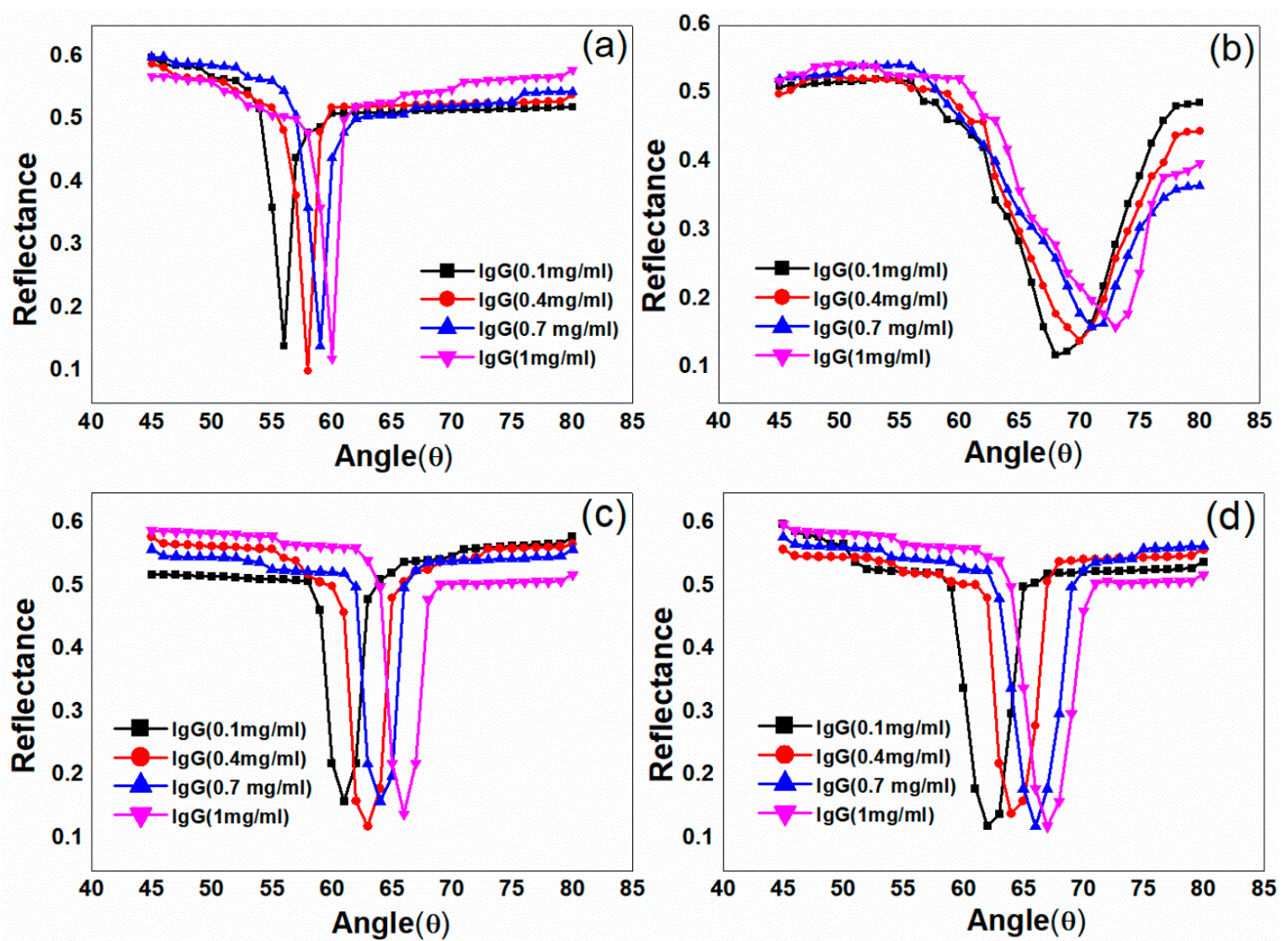

Figure 7. Reflectance vs. incident angle curve of the sensor (a) Ag-I, (b) Au-I, (c) Ag-Au-III, (d) Ag-Au-IV towards different concentrations of protein $(0.1 \mathrm{mg} / \mathrm{mL}, 0.4 \mathrm{mg} / \mathrm{mL}, 0.7 \mathrm{mg} / \mathrm{mL}$, and $1 \mathrm{mg} / \mathrm{mL})$.

Table 4. Experimentally Measured Values of FWHM and Shift in Resonance Angle due to Varied Concentrations of Test Protein.

\begin{tabular}{ccccccccc}
\hline $\begin{array}{c}\text { Concentration of } \\
\text { Protein (IgG) }\end{array}$ & \multicolumn{4}{c}{ FWHM $\left(^{\circ}\right)$} & \multicolumn{3}{c}{ Shift of the Resonance Angle $\left(^{\circ}\right)$} \\
\cline { 2 - 9 } & Ag-I & Au-II & Ag-Au-III & Ag-Au-IV & Ag-I & Au-II & Ag-Au-III & Ag-Au-IV \\
\hline $0.1 \mathrm{mg} / \mathrm{mL}$ & $2 \pm 0.2$ & $10 \pm 0.4$ & $3 \pm 0.2$ & $4.5 \pm 0.3$ & $2 \pm 0.2$ & $4 \pm 0.5$ & $3 \pm 0.3$ & $3.5 \pm 0.4$ \\
\hline $0.4 \mathrm{mg} / \mathrm{mL}$ & $2 \pm 0.2$ & $11 \pm 0.5$ & $3 \pm 0.2$ & $4 \pm 0.4$ & $4 \pm 0.2$ & $6 \pm 0.4$ & $5 \pm 0.2$ & $5.5 \pm 0.2$ \\
\hline $0.7 \mathrm{mg} / \mathrm{mL}$ & $1.5 \pm 0.1$ & $9.5 \pm 0.3$ & $3 \pm 0.1$ & $4.5 \pm 0.4$ & $5 \pm 0.1$ & $7.5 \pm 0.2$ & $6.5 \pm 0.1$ & $7 \pm 0.2$ \\
\hline $1 \mathrm{mg} / \mathrm{mL}$ & $1.5 \pm 0.2$ & $10 \pm 0.3$ & $3 \pm 0.1$ & $4.5 \pm 0.3$ & $6 \pm 0.1$ & $9 \pm 0.2$ & $8 \pm 0.1$ & $8.5 \pm 0.2$ \\
\hline
\end{tabular}

The sensorgrams of the protein detection depicting association and dissociation for the set of four sensors listed in Table 1 is shown in Figure 9. A complete time series measurement cycle consists of two scans of 20 min each: One while the test sample is flown to the surface of the sensor (association phase) and the other while the test sample is flown out of the flow cell (dissociation phase). The baseline is established at the beginning by flowing PBS through the monoclonal anti-IgG functionalized sensor surfaces for $5 \mathrm{~min}$. During the association phase of the sensor, a sensor signal reaches a maxima with the injection of the protein sample. A relatively low concentration $(0.1 \mathrm{mg} / \mathrm{mL})$ target protein is initially exposed to the sensor when the associations of protein results in the red shift of the resonance dip. After $10 \mathrm{~min}$, when the injection of protein is stopped, the sensor produces a steady state level. Later, the sensor signal returns to a value close to the baseline during the dissociation phase when a stream of PBS is passed through the sensor surface. Subsequently, the cycle is repeated by increasing the amount of concentration of the target protein by $0.3 \mathrm{mg} / \mathrm{mL}$, which shows a repeatable behavior in the sensorgrams for each sensor. The dependence of the sensor response on the concentration of protein 
was also noticed. It indicates that the red shift of resonance dip due to the association of protein increases with the increase of the target protein concentration for all the sensors.

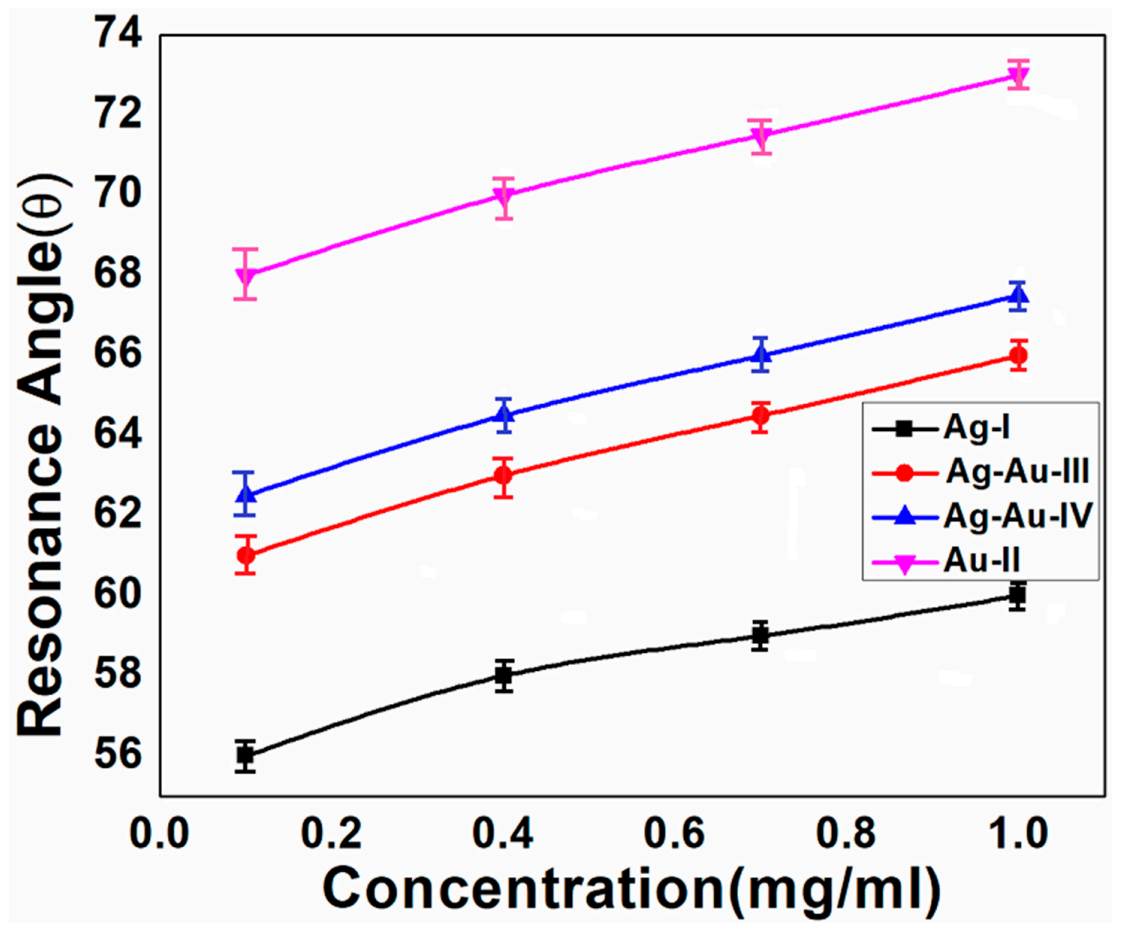

Figure 8. Graph of resonance angle with concentration of antibody protein exposed to sensor surface.
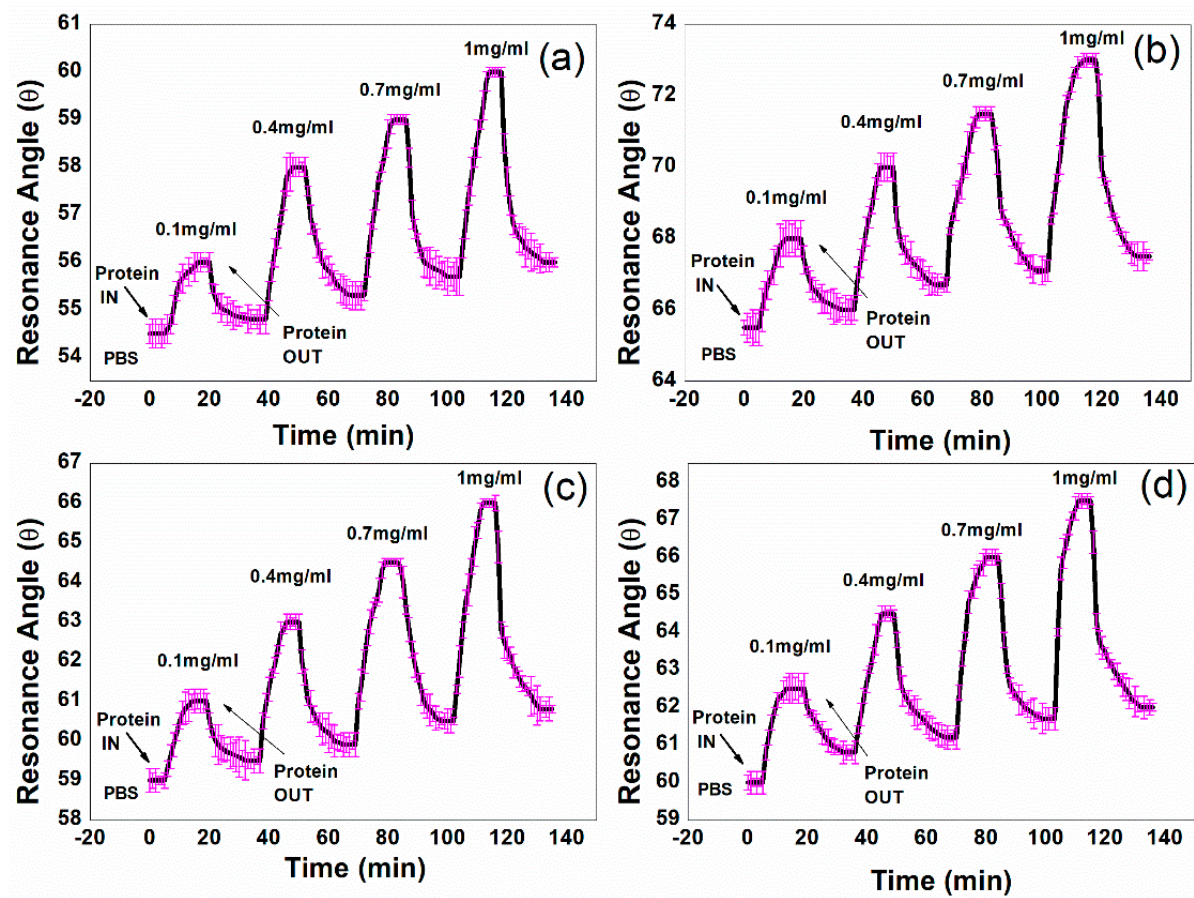

Figure 9. Sensorgrams showing the binding kinetic with protein IgG of different concentrations for sensors: (a) Ag-I, (b) Au-II, (c) Ag-Au-III, (d) Ag-Au-IV.

\section{Conclusions}

This paper reports on the study of a bimetallic surface plasmon resonance (SPR) sensor for antigen-antibody interaction using a custom-made and low-cost portable device. The bimetallic films were prepared using pulsed DC magnetron sputtering of gold over silver which were later 
immobilized with monoclonal anti-IgG in the construction of the SPR biochip. The immobilization of various deposited layers along with the antibody was confirmed by spectroscopic measurements. The performance of the bimetallic sensor chips were investigated in terms of SPR characteristics such as Full Width at Half Maxima (FWHM) and Sensitivity by exposing them to various quantities of the target protein. They demonstrated approximately 3.5-fold reduced values of FWHM compared to pure gold-based sensors indicating better detection accuracy. A significant shift in resonance angle as high as $8.5 \pm 0.2$ due to antigen binding is also displayed by the tested bimetallic sensor which is about 1.42-fold higher compared to pure silver-based sensors. The results confirm the fact that highest sensitivity and smallest FWHM is difficult to be obtained simultaneously for a bimetallic silver-gold SPR sensor because a pure silver-based configuration shows the smallest FWHM while a pure gold based one shows the highest sensitivity. However, a bimetallic SPR sensor with reasonably high sensitivity and small FWHM simultaneously can be obtained experimentally by modification of the film thicknesses of silver-gold in order to meet a tradeoff between the two.

Author Contributions: Conceptualization, B.M.; Formal analysis, R.K., S.C., S.Z., S.S., S.K. and R.D.; Funding acquisition, S.Z.; Investigation, R.K., S.C., S.Z. and B.M.; Methodology, R.K., S.C., S.S., S.K. and R.D.; Supervision, B.M.

Funding: This project has received funding from the European Union's Horizon 2020 research and innovation programme under the Marie Sklodowska-Curie grant agreement No. 798916.

Acknowledgments: The authors are thankful to Arup Ratan Pal, Associate Professor, Institute of Advanced Study in Science and Technology, Guwahati and Sweety Biswasi, Researcher, Institute of Advanced Study in Science and Technology, Guwahati for providing the facilities related to the work. They would also like to thank Khairujjaman Laskar, Department of Chemical Sciences, Tezpur University.

Conflicts of Interest: The authors declare no conflict of interest.

\section{References}

1. Gordon, J.G., II; Ernst, S. Surface Plasmons as a Probe of the Electrochemical Interface. Surf. Sci. 1980, 101, 499-506. [CrossRef]

2. Nylander, C.; Liedberg, B.; Lind, T. Gas Detection by Means of Surface Plasmon Resonance. Sens. Actuators 1982, 3, 79-88. [CrossRef]

3. Liedberg, B.; Nylander, C.; Lundstrom, I. Surface Plasmon Resonance for Gas Detection and Biosensing. Sens. Actuators B Chem. 1983, 4, 299-304. [CrossRef]

4. Liedberg, B.; Nylander, C.; Lundstrom, I. Biosensing with Surface Plasmon Resonance-How it all Started. Biosens. Bioelectron. 1995, 10, 1-9. [CrossRef]

5. Monteiro, J.I.; Mukherji, S.; Kundu, T. Development of a Low-Cost Portable Surface Plasmon Resonance Biosensor. In Proceedings of the IEEE International Conference on Microelectronics, Communication and Renewable Energy, Kanjirapally, Kerala, India, 4-6 June 2013; IEEE: Piscataway, NJ, USA, 2013; p. 989.

6. Mukherji, S.; Hussain, M.I.; Kundu, T.; Chandratre, D. Development of a Surface Plasmon Resonance-Based Biosensing System. In Micro and Smart Devices and Systems; Vinoy, K.J., Ananthasuresh, G.K., Pratap, R., Krupanidhi, S.B., Eds.; Springer: New Delhi, India, 2014; pp. 49-72.

7. Otto, A. Excitation of Nonradiative Surface Plasma Waves in Silver by Method of Frustrated Total Reflection. Z. Fur. Phys. 1968, 216, 398-410. [CrossRef]

8. Kretschmann, E.; Raether, H. Radiative Decay of Non-Radiative Surface Plasmons Excited by Light. Z. Fur Nat. 1968, 23, 2135-2136. [CrossRef]

9. Choudhury, S.N.; Konwar, B.; Kaur, S.; Doley, R.; Mondal, B. Study on Snake Venom Protein-Antibody Interaction by Surface Plasmon Resonance Spectroscopy. Photonic Sens. 2018, 8, 193-202. [CrossRef]

10. Kashyap, R.; Chakraborty, S.; Swarnakar, S.; Humbert, G.; Zeng, S.; Mondal, B. FWHM and Sensitivity Study of Bimetallic SPR Sensor Chip. In Proceedings of the 3rd IEEE International Conference on Electrical, Computer and Communication Technologies (ICECCT), SVS College of Engineering, Coimbatore, Tamil Nadu, India, 20-22 February 2019; IEEE: Piscataway, NJ, USA, 2019; Volume 3, pp. 1682-1685.

11. Homola, J. On the Sensitivity of Surface Plasmon Resonance Sensors with Spectral Interrogation. Sens. Actuators 1997, 41, 207-211. [CrossRef] 
12. Caucheteur, C.; Guo, T.; Albert, J. Review of Plasmonic Fiber Optic Biochemical Sensors: Improving the Limit of Detection. Anal. Bioanal. Chem. 2015, 407, 3883-3897. [CrossRef]

13. Homola, J.; Yee, S.S.; Gauglitz, G. Surface Plasmon Resonance Sensors: Review. Sens. Actuators B Chem. 1999, 54, 3-15. [CrossRef]

14. Nelson, S.G.; Johnston, K.S.; Yee, S.S. High Sensitivity Surface Plasmon Resonance Sensor Based on Phase Detection. Sens. Actuators B Chem. 1996, 35, 187-191. [CrossRef]

15. Maharana, P.K.; Padhy, P.; Jha, R. On the Field Enhancement and Performance of an Ultra-Stable SPR Biosensor Based on Graphene; IEEE Photonics Technology Letters: Piscataway, NJ, USA, 2013; Volume 25, pp. 2156-2159.

16. Hanken, D.G.; Jordan, C.E.; Frey, B.L.; Corn, R.M. Surface Plasmon Resonance Measurements of Ultrathin Organic Films at Electrode Surfaces. In Electroanalytical Chemistry: A Series of Advances; Marcel Dekker: New York, NJ, USA, 1998; Volume 20, pp. 141-225.

17. Chen, X.; Jiang, K. Effect of Aging on Optical Properties of Bimetallic Sensor Chips. Opt. Soc. Am. 2010, 18, 1105-1112. [CrossRef] [PubMed]

18. Zynio, S.A.; Samoylov, A.V.; Surovtseva, E.R.; Mirsky, V.M.; Shirshov, Y.M. Bimetallic Layers Increase Sensitivity of Affinity Sensors Based on Surface Plasmon Resonance. Sensors 2002, 2, 62-70. [CrossRef]

19. Yuan, X.C.; Ong, B.H.; Tan, Y.G.; Zhang, D.W.; Irawan, R.; Tjin, S.C. Sensitivity-Stability-Optimized Surface Plasmon Resonance Sensing with Double Metal Layers. J. Opt. A Pure Appl. Opt. 2006, 8, 959-963. [CrossRef]

20. Ong, B.H.; Yuan, X.; Tjin, S.C.; Zhang, J.; Ng, H.M. Optimised Film Thickness for Maximum Evanescent Field Enhancement of a Bimetallic Film Surface Plasmon Resonance Biosensor. Sens. Actuators B Chem. 2006, 114, 1028-1034. [CrossRef]

21. Wu, S.Y.; Ho, P.H. Sensitivity improvement of the surface plasmon resonance optical sensor by using a gold-silver transducing layer. In Proceedings of the 2002 IEEE Hong Kong Electron Devices Meeting, Hong Kong, China, 22 June 2002; pp. 63-68.

22. Chen, Y.; Zheng, R.; Zhang, D.; Lu, Y.; Wang, P.; Ming, H.; Luo, Z.; Kan, Q. Bimetallic Chips for a Surface Plasmon Resonance Instrument. Appl. Opt. 2017, 50, 387-391. [CrossRef]

23. Huang, Y.; Xia, L.; Wei, W.; Chuang, C.J.; Du, C. Theoretical Investigation of Voltage Sensitivity Enhancement for Surface Plasmon Resonance Based Optical Fiber Sensor with a Bimetallic Layer. Opt. Commun. 2014, 333, 146-150. [CrossRef]

24. Chen, S.; Lin, C. High-Performance Bimetallic Film Surface Plasmon Resonance Sensor Based on Film Thickness Optimization. Optik 2016, 127, 7514-7519. [CrossRef]

25. Nimse, S.B.; Song, K.; Sonawane, M.D.; Sayyed, D.R.; Kim, T. Immobilization Techniques for Microarray: Challenges and Applications. Sensors 2014, 14, 22208-22229. [CrossRef]

26. Rusmini, F.; Zhong, Z.; Feijen, J. Protein Immobilization Strategies for Protein Biochis. Biomacromolecules 2007, 8, 1775-1789. [CrossRef]

27. Lee, J.E.; Seo, J.H.; Kim, C.S.; Kwon, Y.; Ha, J.H.; Choi, S.S.; Cha, H.J. A Comparative Study on Antibody Immobilization Strategies onto Solid Surface. Korean J. Chem. Eng. 2013, 30, 1934-1938. [CrossRef]

28. Hodneland, C.D.; Lee, Y.S.; Min, D.H.; Mrksich, M. Selective Immobilization of Proteins to Self-Assembled Monolayers Presenting Active Site-Directed Capture Ligands. Proc. Natl. Acad. Sci. USA 2002, 99, 5048-5052. [CrossRef]

29. Peters, R.F.; Gutierrez-Rivera, L.; Dew, S.K.; Stepanova, M. Surface Enhanced Raman Spectroscopy Detection of Biomolecules Using EBL Fabricated Nanostructured Substrates. J. Vis. Exp. Jove 2015, 2015, e52712. [CrossRef] [PubMed]

30. Mohapatra, S.; Moirangthem, R.S. Theoretical Study of Modulated Multi-Layer SPR Device for Improved Refractive Index Sensing. IOP Conf. Ser. Mater. Sci. Eng. 2018, 310, 012017. [CrossRef]

31. Kuo Chih, C.; Lin, C.Y.; Chen, Y.D.; Shean Jen, C. Optimizing Silver Film for Surface Plasmon Coupled Emission Induced Two-Photon Excited Fluorescence Imaging. Opt. Express 2011, 19, 5386-5396.

32. Whiteside Paul, J.D.; Chininis Jeffrey, A.; Hunt Heather, K. Techniques and Challenges for Characterizing Metal Thin Films with Applications in Photonics. Coat. Rev. 2016, 6, 35. [CrossRef]

33. Aslan, K.; Luhrs, C.C.; Perez-Luna, V.H. Controlled and Reversible Aggregation of Biotinylated Gold Nanoparticles with Streptavidin. J. Phys. Chem. B 2004, 108, 15631-15639. [CrossRef] 
34. Weng, G.; Li, J.; Zhoa, J. Enhanced Resonance Light Scattering of Antibody Covalently Conjugated Gold Nanoparticles due to Antigen-Antibody Interaction Induced Aggregation. Nanosci. Nanotechnol. Lett. 2013, 5, 1-7. [CrossRef]

35. Sharma Anuj, K.; JhaRajan Gupta, B.D. Fiber-OPTIC Sensors Based on Surface Plasmon Resonance: A Comprehensive Review. IEEE Sens. 2007, 7, 1118-1129. [CrossRef] 